\section{BMJ Open Respiratory Research}

\title{
Initial experience with transbronchial cryoablation as a novel local treatment for malignant peripheral lung lesions
}

\author{
Mitsutomo Kohno, ${ }^{1}$ Ryo Hashimoto, ${ }^{1}$ Kana Oiwa, ${ }^{1}$ Hideki Yashiro, ${ }^{2}$ \\ Seishi Nakatsuka, ${ }^{2}$ Masafumi Kawamura, ${ }^{3}$ Masayuki Iwazaki ${ }^{1}$
}

To cite: Kohno M, Hashimoto R, Oiwa K, et al. Initial experience with transbronchial cryoablation as a novel local treatment for malignant peripheral lung lesions. BMJ Open Resp Res 2018;5:e000315. doi:10.1136/ bmjresp-2018-000315

Received 17 May 2018 Revised 29 August 2018 Accepted 31 August 2018

\section{Check for updates}

${ }^{1}$ Division of General Thoracic Surgery, Department of Surgery, Tokai University School of Medicine, Isehara, Japan

${ }^{2}$ Department of Diagnostic Radiology, Keio University School of Medicine, Tokyo, Japan

${ }^{3}$ Department of General Thoracic Surgery, Teikyo University School of Medicine, Tokyo, Japan

Correspondence to Dr Mitsutomo Kohno; kohno@tokai.ac.jp

\section{ABSTRACT}

Introduction Percutaneous cryoablation has been adopted for small, malignant peripheral pulmonary lesions and has yielded favourable results as a less invasive local treatment. A transbronchial approach may reduce the risks of complications, such as pneumothorax and pleural haemorrhage. A fundamental animal experiment on transbronchial cryoablation was performed to examine its immediate safety and effectiveness.

Methods Experimental cryoablation was performed on swine lungs using a rigid cryoprobe, $2.4 \mathrm{~mm}$ in diameter. The probe was introduced from the right main bronchus into the distal bronchus via thoracotomy, perforated the end of the conducting bronchus and reached the lung parenchyma. The temperature of the cryoprobe tip reaches approximately $-130^{\circ} \mathrm{C}$ during freezing and approximately $20^{\circ} \mathrm{C}$ during thawing. After three freeze-thaw cycles, the cryoprobe was removed.

Results No significant haemorrhage was bronchoscopically observed in the airways throughout the experiment. The chronological changes and spatial distribution of the temperature of the pulmonary tissue circumferential to the point of the cryoprobe were similar to those seen with transpleural cryoablation and the less than $-20^{\circ} \mathrm{C}$ thermal zone seemed to be established within a radius of at least $12 \mathrm{~mm}$. The central destruction zone of alveolar structures was histologically similar to the thermal zone, while the conducting bronchus structure and the accompanying pulmonary artery were not severely affected.

Conclusion Experimental transbronchial cryoablation with a rigid cryoprobe could effectively freeze and destroy peripheral lung alveoli without any significant immediate adverse effects. This may suggest the potential clinical application of transbronchial cryoablation for peripheral malignant lung lesions.

\section{INTRODUCTION}

Cryoablation therapy is one non-surgical local treatment to control malignant tumours. A tumour is frozen using a specific cryoprobe that is inserted percutaneously into the tumour under CT, MRI or ultrasound guidance. We have adopted cryoablation for lung malignant tumours beginning in 2002 and previously reported satisfactory results

\section{Key messages}

Transbronchial cryoablation could become a local treatment modality for small peripheral lung malignant lesions, avoiding most complications of percutaneous cryoablation, such as pneumothorax and pleural effusion.

- Experimental transbronchial cryoablation with a rigid cryoprobe confirmed extension of the less than $-20^{\circ} \mathrm{C}$ thermal zone and caused neither pleural defects nor haemorrhage.

- For clinical application, development of a new flexible cryoprobe that can be introduced into the peripheral lung via a conducting narrow bronchus under bronchoscopic guidance is necessary.

of percutaneous cryoablation for metastatic lung tumours and early stage primary lung cancers. ${ }^{1}$ We have obtained equivalent treatment results for tumours smaller than $3 \mathrm{~cm}$ with stereotactic radiotherapy or radiofrequency ablation..$^{2-4}$ The 3-year local control rate, 3-year disease-free survival rate and 3-year overall survival rate of patients with stage IA primary lung cancer after cryoablation were $97 \%, 88 \%$ and $67 \%$ respectively, and average overall survival time was 62 months. ${ }^{5}$ Because it can be performed under local anaesthesia, and the loss of pulmonary function after cryoablation may be minimal compared with surgical resection and irradiation, ${ }^{3}$ it is a less invasive treatment for lung malignancy. Treatment for multiple lesions or repeated treatments is possible. Its usefulness has been accepted worldwide. ${ }^{67}$

There are some problems to be resolved with percutaneous cryoablation therapy, which are caused by penetration of the chest wall and parietal and visceral pleura. ${ }^{78}$ These complications include pneumothorax, pleural effusion, haemothorax and haemoptysis; additionally, pyothorax and dissemination of malignant tumours can occur. The most frequent complication is pneumothorax, which occurs in $62 \%$ of patients who 


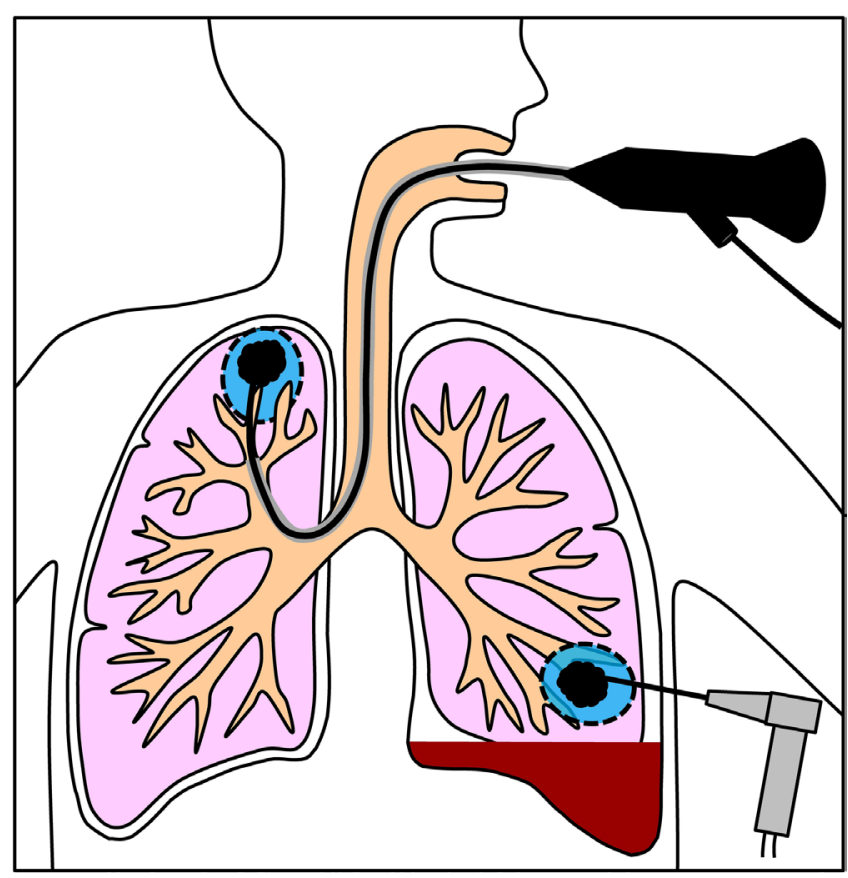

Figure 1 Schematic representation of cryoablation for peripheral pulmonary malignancy via transbronchial approach in the right lung and percutaneous approach in the left lung. Transbronchial insertion of a cryoprobe avoids penetration of the chest wall and the parietal and visceral pleura and thereby would reduce the risk of pneumothorax, haemothorax and dissemination of malignant tumours.

undergo percutaneous cryoablation. ${ }^{8}$ The tract through the chest wall usually does not lead to pneumothorax; however, the tract in the visceral pleura fails to prevent air leak; injections, including fibrin glue, into the probe tract have been used to avoid this complication. Some patients require thoracic tube drainage for pneumothorax, and prolonged air leakage may lead to deterioration in respiratory function. Haemothorax can be due to injury of the intercostal artery or vein. Massive haemoptysis is also possible, caused by injury of pulmonary arteries or veins. The most important problem is the risk of pleural dissemination of malignant tumours. Insertion and removal of the cryoprobe may disseminate malignant cells, although this is very rare. ${ }^{9}$

We consider that these problems would be resolved if the cryoablation is performed transbronchially rather than percutaneously (figure 1). The transbronchial approach does not require penetration of the chest wall and pleura; thus the risk of pneumothorax will be decreased. Bleeding from intercostal arteries and veins would not occur and haemothorax and massive haemoptysis might be prevented. The risk of pleural dissemination of malignant tumours would also be reduced. There have been some studies concerning cryotherapy for endobronchial lesions. However, so far there is no experimental study evaluating transbronchial cryoablation for peripheral pulmonary lesions. The purpose of the present study is to investigate the immediate safety and freezing effect of transbronchial cryoablation in animals.
Materials and methods

The investigation conformed to The Guide for the Care and Use of Laboratory Animals published by the U.S. National Institutes of Health (NIH Publication No. 85-23, revised 1996). All procedures described in this report were approved by the Animal Experimentation Committee of Tokai University.

\section{Animal preparation}

We used three specific-pathogen free, female domestic pigs, 3 months of age, weighing $40 \mathrm{~kg}$. They were anaesthetised with an intramuscular injection of medetomidine $20 \mu \mathrm{g} / \mathrm{kg}$ and midazolam $0.3 \mathrm{mg} / \mathrm{kg}$, intubated with a double-lumen endotracheal tube and mechanically ventilated at a respiratory rate of 15-20 breaths/ min with a tidal volume of $10 \mathrm{~mL} / \mathrm{kg}$, a positive end-expiratory pressure of $5.0 \mathrm{~cm} \mathrm{H}_{2} \mathrm{O}$ and fraction of inspired oxygen of 1.0. Anaesthesia was maintained by inhalation of sevoflurane $(3 \%-5 \%)$ and an intravenous injection of rocuronium bromide $(0.4 \mathrm{mg} / \mathrm{kg} /$ hour$)$. Each pig was placed in the left decubitus position and underwent a right thoracotomy. The cranial, medial and caudal lobe bronchi were exposed.

\section{System of cryoablation}

The freezing and thawing by the cryoprobe is based on the Joule-Thomson effect of the rapid expansion of high-pressure argon and helium for freezing and thawing, respectively. The probe is comprised of an inlet tube and a closed-ended outer tube disposed coaxially around the inlet tube. The gas is injected from the inlet tube with high pressure inside of the tip of the outer tube, and the temperature of the probe surface reaches approximately $-130^{\circ} \mathrm{C}$ during freezing and approximately $40^{\circ} \mathrm{C}$ during thawing. Because there is no available flexible cryoprobe specific for transbronchial cryoablation, a rigid $2.4 \mathrm{~mm}$ cryoprobe for percutaneous cryoablation (figure 2A) and cryosurgical unit were used (CRYO-care Cryosurgical Unit; Endocare, Irvine, California, USA). A probe contains a $4.5 \mathrm{~cm}$-long heat exchanger in the tip, and an ice ball of axisymmetric drop shape is made around the probe tip within a few minutes after the start of argon gas injection, when the cryoprobe is cooled down in water as a test operation (figure $2 \mathrm{~B}$ ).

\section{Transbronchial introduction of a rigid cryoprobe}

Figure 3A illustrates transbronchial insertion of a rigid cryoprobe. The cryoprobe was introduced in the lung using an 8-Fr introducer sheath $(10 \mathrm{~cm}$ in length, $\varphi 2.78$ mm I.D., Radifocus Introducer II H), the tapering part of which was cut so that the tip of the cryoprobe could be exposed up to $4.5 \mathrm{~cm}$ in length (figure $2 \mathrm{~A}$ ). After the right lung was inflated at an airway pressure of $20 \mathrm{~cm}$ $\mathrm{H}_{2} \mathrm{O}$, an introducer sheath was introduced via the right main bronchus into the distal bronchus using an accessary guidewire and dilator. After the dilator was removed, 

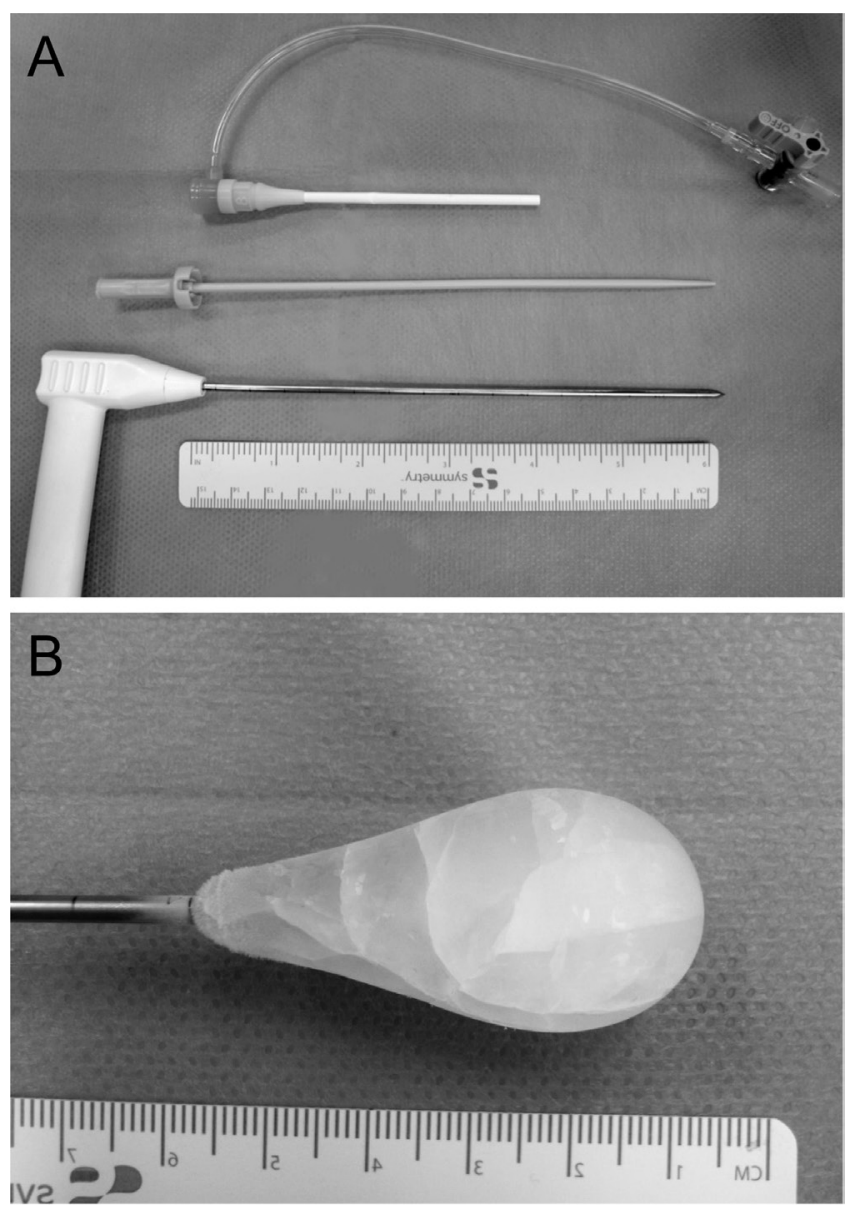

Figure 2 A rigid $2.4 \mathrm{~mm}$ cryoprobe (CRYO-care cryosurgical unit; Endocare, Irvine, California, USA) and an 8-Fr introducer sheath $(10 \mathrm{~cm}$ in length, $\varphi 2.78 \mathrm{~mm}$ I.D., Radifocus introducer IIH, Terumo, Japan), the tapering part of which was cut to expose the heat exchanger of the cryoprobe up to $4.5 \mathrm{~cm}$ in length and an accessory dilator.

a cryoprobe was introduced into the sheath, perforated the end of a conducting bronchus and the tip of the cryoprobe was inserted into the targeted lung parenchyma (figure 3B). As a control group mimicking percutaneous cryoablation, the cryoprobe was inserted transpleurally into the targeted lung areas. In another group, the cryoprobe was introduced transbronchially but did not perforate the conducting bronchus; that is, the cryoprobe loosened in the proximal site of the conducting bronchus. Adverse effects, such as bleeding or airway injury, were examined by bronchoscopy after the insertion of the cryoprobe and throughout the cryoablation procedures. Blood pressure, heart rate and percutaneous arterial oxygen saturation were monitored throughout the experiment.

\section{Cryoablation procedure and temperature measurement}

Three freeze-thaw cycles were conducted, as previously described. ${ }^{10}$ This is the same protocol used in the clinical setting at our institution. For the freeze-thaw cycle, the first freezing time was $5 \mathrm{~min}$ for preparation and the second

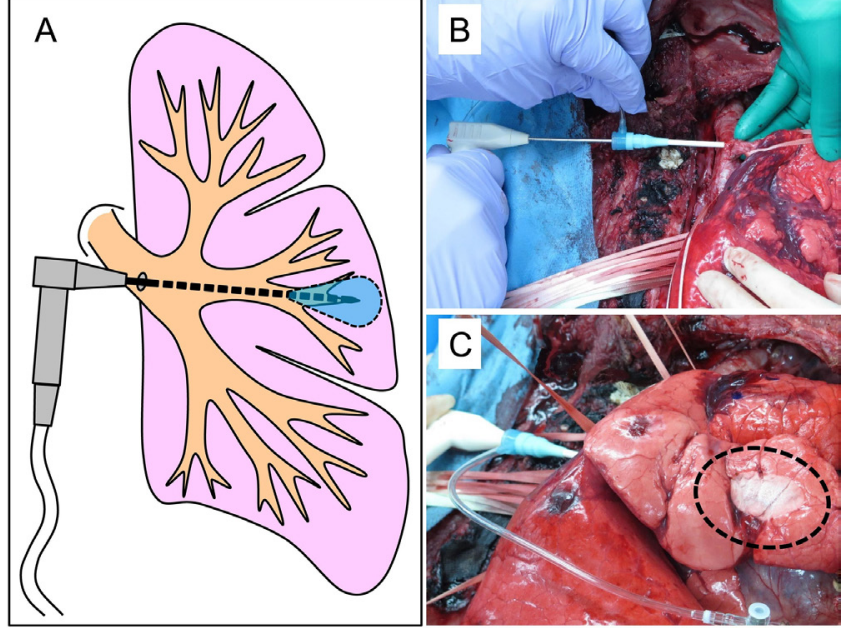

Figure 3 An illustration of experimental transbronchial cryoablation with a rigid cryoprobe $(A)$ and photographs of transbronchial introduction of a cryoprobe $(\mathrm{B})$ and cryoablation $(\mathrm{C})$ on a pig. A rigid cryoprobe is inserted from the right main bronchus into a distal bronchus through an introducer $(\mathrm{B})$. In the freezing phase, the temperature of the probe tip falls approximately $-130^{\circ} \mathrm{C}$ and the pleura on the frozen lung appears focally whitish with frost (within circle of dashed line) (C).

and third freezing periods were $10 \mathrm{~min}$ each. For the first and second thaw periods, active thawing was performed until the temperature reached $20^{\circ} \mathrm{C}$. The helium gas was then stopped, and passive thawing was allowed until the total thawing time reached $10 \mathrm{~min}$. For the third thaw period, high-pressure helium gas was used until the cryoprobe and the guiding sheath were removed. Type K thermocouples were used for temperature measurement as previously described. ${ }^{10}$ Temperature was measured simultaneously at four points every one second using a data logger (Pico Technology, Cambridgeshire, UK). A $2.4 \mathrm{~mm}$ cryoprobe was inserted at the centre of a targeted lung area, and four thermocouples were inserted in the lung on the plane including the needle point and at right angles to the major axis of the cryoprobe, at locations (1) adjacent to the needle point, (2) $6 \mathrm{~mm}$, (3) $12 \mathrm{~mm}$ and (4) $18 \mathrm{~mm}$ from the needle point, respectively (figure 4). Isothermal zones around a probe tip would be formed in the plane as previously reported. ${ }^{11}$ Temperature of the cryoprobe itself is also recorded simultaneously with another thermocouple equipped in the cryoprobe.

\section{Histological examination}

All pigs were euthanised after three freeze-thaw cycles and temperature measurement, and the lungs were resected for histological examination. The lungs were inflated with transbronchial injection of $10 \%$ buffered formalin at a pressure of $20 \mathrm{cmH}_{2} \mathrm{O}$, fixed, embedded in paraffin, cut into 4- $\mu$ m-thick slices and stained with H\&E. Histological changes of the lung parenchyma, bronchi and vessels after cryoablation were examined in sections perpendicular to the major axis of the cryoprobe tract 
Places of thermocouples:

$-18 \mathrm{~mm},-12 \mathrm{~mm},-6 \mathrm{~mm}$ away from point of cryoprobe,

$-0 \mathrm{~mm}$, adjacent to probe, $\quad-$ inside probe.
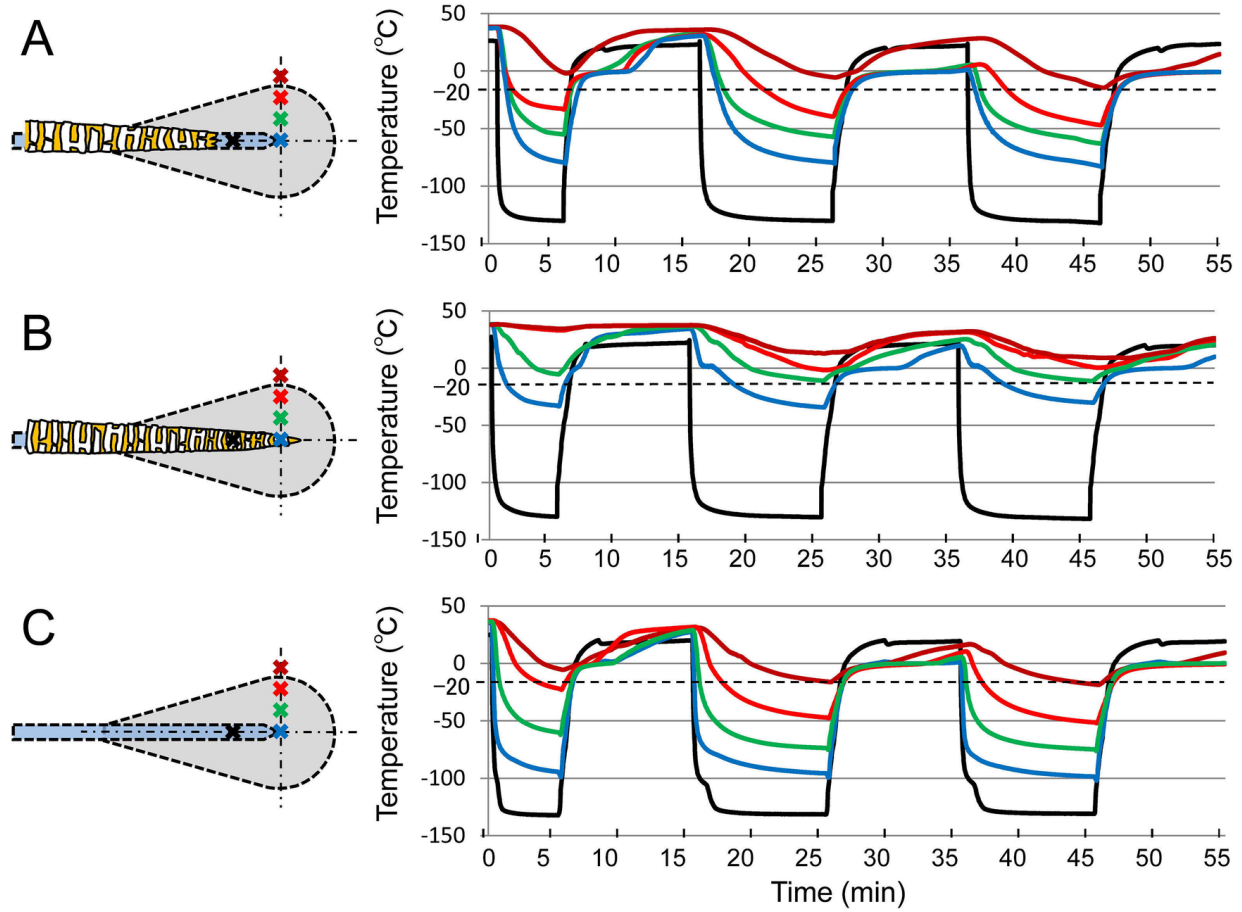

Figure 4 Illustrated positional relationship of the cryoprobe and thermocouples, and typical chronological temperature changes of targeted lung tissue areas according to the approach of the cryoprobe, during transbronchial cryoablation with the cryoprobe perforating the end of a conducting bronchus $(A)$; insufficient transbronchial cryoablation with the cryoprobe not perforating the end of a conducting bronchus $(\mathrm{B})$ and transpleural cryoablation mimicking percutaneous cryoablation (C) in pigs. Thermocouples were placed on a plane including the cryoprobe point and at a right angle to the long axis of the cryoprobe. The temperatures according to the distance from the cryoprobe point are described by colours: blue for $0 \mathrm{~mm}$ (adjacent to the point), green for $6 \mathrm{~mm}$, red for $12 \mathrm{~mm}$ and brown for $18 \mathrm{~mm}$. The temperature of the cryoprobe itself is shown in black.

and including sections through the major axis of the tract.

\section{Statistical analysis}

The data are presented as means \pm standard deviations. Between group differences were examined by Fisher's Protected Least Significant Difference test. $\mathrm{P}<0.05$ was considered statistically significant. The data were analysed using StatView-J 5.0 (Abacus Concepts, Berkeley, California, USA)

\section{RESULTS}

Oxygenation and haemodynamics during experimental transbronchial cryoablation of pig lungs

Percutaneous arterial oxygen saturation of every pig was $98 \%-100 \%$ before the cryoablation procedure, and was stable at $98 \%-100 \%$ throughout the experiment under mechanical ventilation. Haemodynamics were also stable, with systolic blood pressure in the range of 80-105 $\mathrm{mm} \mathrm{Hg}$ and heart rate of $60-75$ beats per min during the experiments.
Transbronchial insertion of a cryoprobe into peripheral lung Transbronchial perforation of a conducting bronchus and insertion into the peripheral lung with a $2.4 \mathrm{~mm}$ cryoprobe did not cause significant endobronchial haemorrhage, as examined with bronchoscopy. A radiograph shows no infiltration around the cryoprobe transbronchially inserted into the lung parenchyma (figure 5). There was also no endobronchial haemorrhage observed after every procedure throughout the three freeze-thaw cycles. Only minimal blood was seen in the segmental bronchi after removal of the cryoprobe.

\section{Spatial temperature distribution and chronological} temperature changes of lung tissues during freezing and thawing cycles

The typical chronological temperature changes and the minimum temperatures of targeted lung tissue areas are shown according to the approach of the cryoprobe and according to the distance from the point of the cryoprobe in figure 5 and table 1 . Regardless of the approach, in the freezing phase, the temperature of a cryoprobe 


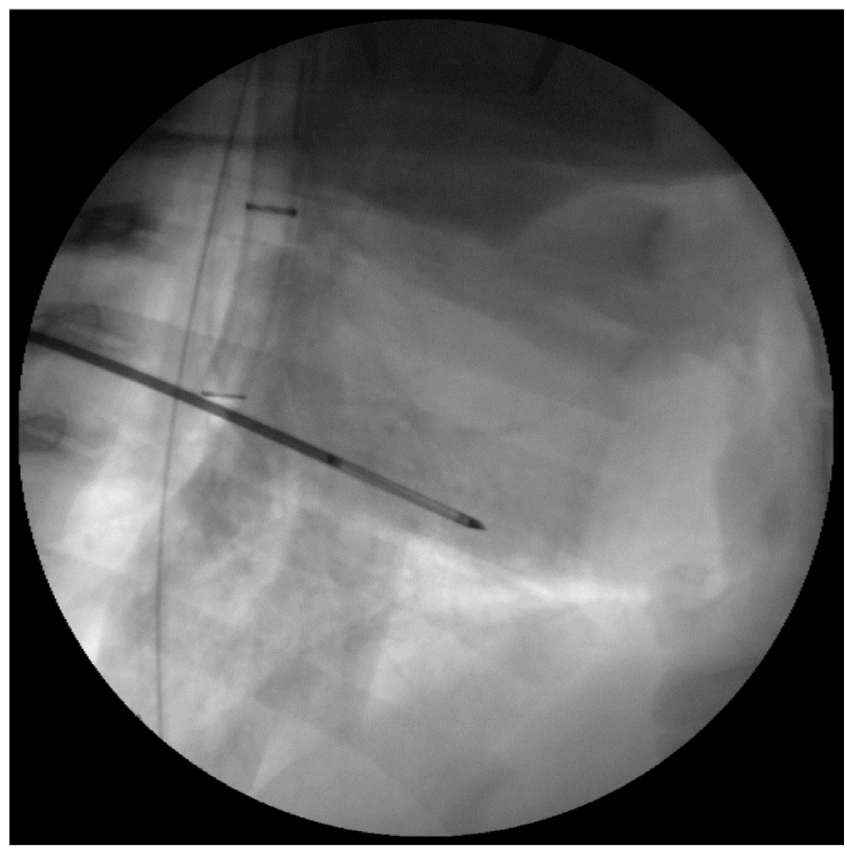

Figure 5 Lateral view of chest roentgenogram showing a cryoprobe transbronchially introduced into the lung periphery in a pig. No significant infiltration is seen around the probe after transbronchial insertion.

itself immediately fell to $-130^{\circ} \mathrm{C}$ within approximately 2 min after the start of argon gas application, and the temperature was maintained during argon gas exposure for 5 or $10 \mathrm{~min}$. During the freezing phase, the surface of targeted lungs is cooled, and there was visible frost (figure 3C). In the thawing phase, the temperature of the cryoprobe immediately increased up to $20^{\circ} \mathrm{C}$ within approximately 2 min after the start of helium gas application, and the temperature was maintained until the next freezing phase. When a cryoprobe tip perforated the end of the conducting bronchus, the tip containing a heat exchanger was exposed within the lung parenchyma or contacted firmly the inner walls of the narrow bronchus (figure 4A). The temperature of the lung tissue area adjacent to the probe point and at areas $6 \mathrm{~mm}$ and $12 \mathrm{~mm}$ aside from the probe point decreased drastically after the temperature of the probe fell, whereas the temperature drop in the area $18 \mathrm{~mm}$ from the point was delayed. The drop in temperature of lung tissue was remarkable as it approached the probe. After $5 \mathrm{~min}$ of the first freezing, the temperature of lung tissues at the four points simultaneously increased as the temperature of a probe tip rapidly increased and reached approximately $30^{\circ} \mathrm{C}$ at the end of the first thawing phase. In the second freezing phase, the temperature changes were similar to the first phase, with the lowest temperature of each area maintained for a longer time than during the first phase and minimum temperatures at the end of the second freezing phase slightly lower than those at the end of the first phase. In the second thawing phase, the temperatures other than the area adjacent to the point remained low, at approximately $0^{\circ} \mathrm{C}$, until the end of the second thawing phase. In the third freezing and thawing phase, temperature changes were similar to the second phase, with the minimum temperatures of each area recorded at the end of the third freezing phase (table 1). On the other hand, when the cryoprobe did not perforate the conducting bronchus and loosened within the bronchus, the temperatures of each area were shifted significantly higher compared with the other groups and only the area adjacent to the probe point fell below $-20^{\circ} \mathrm{C}$ (figure 4B). When the cryoprobe was inserted transpleurally, mimicking conventional percutaneous cryoablation, the temperature changes of each area were distributed according to the distance between the probe point and the lung tissue similar to those recorded when the probe was transbronchially inserted and perforated the end of the conducting bronchus.

\section{Histology of the lungs after transbronchial cryoablation}

The lung specimens, which were obtained after transbronchial cryoablation with the cryoprobe perforating the end of the conducting bronchus, were cut into slices of the cryoprobe tract including the major axis of the tract. In sections perpendicular to the major axis, the alveolar structure was destroyed and extensive haemorrhage was dominant in the central area, with the tract

Table 1 Minimum temperature $\left({ }^{\circ} \mathrm{C}\right)$ of the lung tissue in freezing phase of experimental cryoablation according to the approach of a cryoprobe and according to distance from a point of the probe

\begin{tabular}{llcl}
\hline & \multicolumn{2}{l}{ Transbronchial approach } & \\
\cline { 2 - 4 } $\begin{array}{l}\text { Distance between probe } \\
\text { points and thermocouples }\end{array}$ & \multicolumn{2}{l}{ Perforation of a conducting bronchus with a cryoprobe } & Transpleural approach \\
\cline { 2 - 4 } & Yes & No & $-13.5 \pm 5.7$ \\
\hline $18 \mathrm{~mm}$ & $-10.3 \pm 3.7$ & $8.8 \pm 16.7^{\star}$ & $-40.9 \pm 12.9$ \\
$\sim 12 \mathrm{~mm}$ & $-44.3 \pm 3.3$ & $-9.19 \pm 2.7^{\star}$ & $-71.2 \pm 6.4$ \\
$\sim 6 \mathrm{~mm}$ & $-56.4 \pm 4.9$ & $-32.7 \pm 1.7^{\star}$ & $-100.0 \pm 1.3$ \\
$0 \mathrm{~mm}$ & $-77.9 \pm 5.9$ & $-130.6 \pm 0.8$ & $-131.5 \pm 0.5$ \\
\hline
\end{tabular}

Values are means $\pm S D(n=3)$ in each measurement.

" $\mathrm{P}<0.05$ vs the other groups (Fisher's PLSD test).

PLSD, Protected Least Significant Difference. 


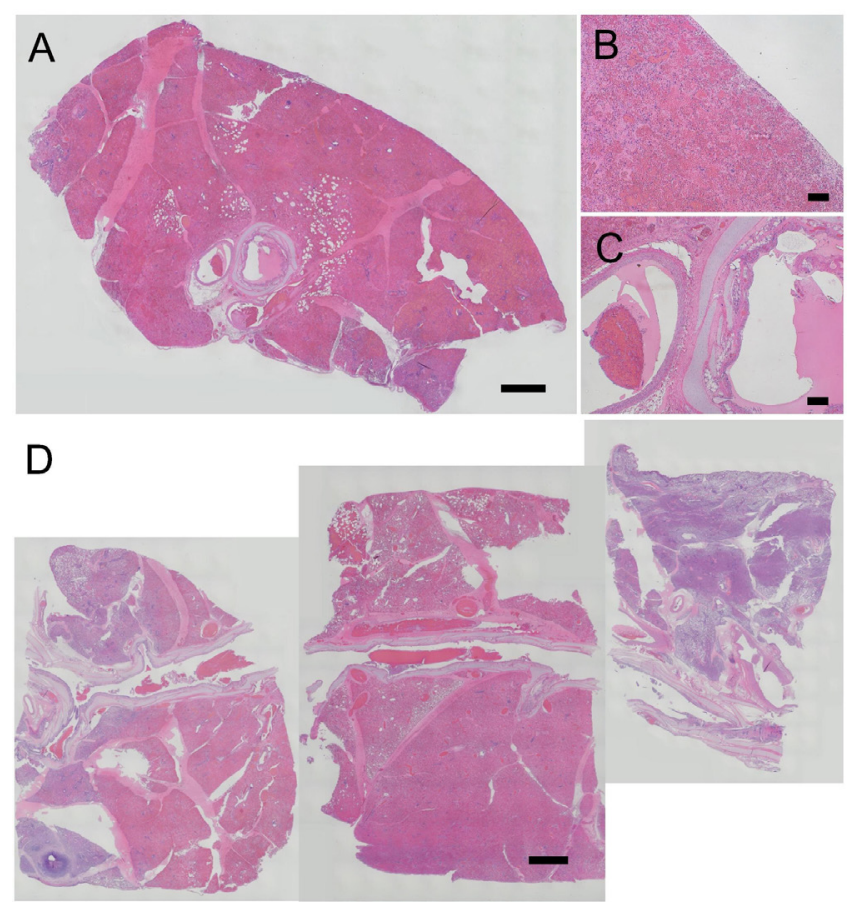

Figure 6 Representative histological sections of porcine lungs obtained after transbronchial cryoablation. A composite histological photograph shows a transverse section through the axis of a bronchus in which a cryoprobe was inserted (A) (bar=2 $\mathrm{mm}$ ). High power fields show alveoli and pleura in the destruction zone $(B)$ and the bronchus and an accompanying pulmonary artery $(C)$ after transbronchial cryoablation (bar=100 $\mu \mathrm{m}$ ). Composite histological Photographs of porcine lung sections including the major axis of a cryoprobe tract after transbronchial cryoablation showing a reddish destruction zone along a bronchus (D) (bar=2 mm).

as its centre, up to a radius of approximately $15 \mathrm{~mm}$; in this area, the tissue appeared reddish, whereas the visceral pleura covering the alveolar destruction zone did not break down (figure 6A,B). The narrow bronchus was expanded slightly compared with the accompanying pulmonary artery and ciliated columnar epithelium disappeared from the inner wall of the bronchus, while the hyaline cartilage plates and bronchial adventitia maintained their structures (figure 6C). The pulmonary artery accompanying the bronchus maintained its structures, including the intima, media and adventitia (figure 6C). In the sections of the plane including the long axis of the conducting bronchus, small blood clot was seen within the bronchus. The destruction of the alveolar structure spread symmetrically along the expanded bronchus, while the conducting bronchus and accompanying pulmonary artery maintained their structures (figure 6D).

\section{DISCUSSION}

Experimental transbronchial cryoablation with a rigid cryoprobe was attempted on porcine peripheral lungs for the first time. It caused neither pleural defects, as seen in transpleural cryoablation, nor haemorrhage. The chronological changes and spatial distribution of temperature in the lung tissues circumferential to the cryoprobe tip during freeze and thaw cycles were similar to those produced during transpleural cryoablation, mimicking conventional percutaneous cryoablation within the lung parenchyma, and the histological expanse of alveolar destruction after cryoablation was observed. No significant immediate adverse effects, such as airway haemorrhage, were identified. Transbronchial cryoablation may represent a minimally invasive technique for small malignant lung lesions in the peripheral lung, such as early stage primary lung cancers or metastatic malignant lung tumours. For clinical application, development of a new flexible cryoprobe that can be introduced into the peripheral lung via a conducting narrow bronchus under bronchoscopic guidance is necessary.

We previously compared experimental findings of transpleural cryoablation with clinical CT findings of percutaneous cryoablation and extrapolated to local cancer control outcomes. We reported that the less than $-20^{\circ} \mathrm{C}$ thermal zone, which was the most central zone surrounding the cryoprobe, corresponded to the histological zone of complete destruction, and that the 3-year local cancer control rate of nodules contained within the central zone was $82 \%$, while that of nodules outside this zone was $33 \% .^{11}$ Therefore, in the present study of transbronchial cryoablation in the peripheral lungs, we think it is very important that the $-20^{\circ} \mathrm{C}$ thermal zone is created in the same manner as transpleural cryoablation.

Chronological temperature changes of lung parenchyma with the cryoprobe inserted transbronchially and perforating the conducting bronchus were similar to those frozen with the cryoprobe inserted transpleurally. The temperature of lung parenchyma circumferential to the cryoprobe followed that of the cryoprobe, slightly later in the freezing and thawing phases. Spatial temperature changes of transbronchial ablation seemed to be distributed concentrically around the circular tip of the cryoprobe, which is similar to the effect of transpleural ablation. The less than $-20^{\circ} \mathrm{C}$ thermal zone seemed to be established in the range of a radius of at least $12 \mathrm{~mm}$, which was also confirmed histologically. The temperature of lung parenchyma at $12 \mathrm{~mm}$ from the probe tip tended decrease with the second or third, rather than the first, freeze cycle in both transbronchial and transpleural cryoablation. This suggests extension of the less than $-20^{\circ} \mathrm{C}$ thermal zone during the second and third freezing phase compared with the first phase. It is known that water leakage after the first freezing and thawing phase promotes the freezing effect in the circumference of the freeze area, because water has a high thermal conductivity. On the other hand, extension of the less than $-20^{\circ} \mathrm{C}$ thermal zone seemed to be limited when the cryoprobe did not perforate the conducting bronchus and was loosened within it. The existence of air in the bronchus would significantly inhibit freezing, because the thermal conductivity of the air is less than $1 / 20$ th that of water. 
We histologically estimated the radius of the central destruction zone to be approximately $15 \mathrm{~mm}$ after experimental transbronchial cryoablation in the present setting, using a $2.4 \mathrm{~mm}$ cryoprobe and cryosystem employing argon gas. This seems compatible with the results of temperature measurements, that is, the extension of the $-20^{\circ} \mathrm{C}$ thermal zone. This suggests that transbronchial cryoablation has the potential to provide local control of cancers similar to percutaneous cryoablation in the clinical settings. However, the destruction zone was limited when the cryoprobe was loosened within the bronchus, which was compatible with the results of temperature measurement showing limited spatial temperature distribution. Histological examination also revealed that acute effects of transbronchial ablation on bronchial structures were minimal, although only the perforated end of the conducting bronchus was expanded.

There are some limitations in the present experiment. First, a rigid cryoprobe was used and thoracotomy was required to introduce the probe into the distal airways. The only potentially suitable approach for transbronchial cryoablation using a conventional rigid cryoprobe might be considered the use of a rigid bronchoscopy. This approach would allow direct access to the lower and, to some extent, middle lobes, although it would not permit access to the upper lobes. The cryoprobe required for transbronchial cryoablation will be discussed later. Second, we did not freeze tumour, but normal lung tissue. However, tumour tissue is easier to freeze as compared with normal lung tissue, because heat conductivity of tumour tissue, containing a higher water content, is greater than that of lung tissue, containing more air. Therefore, we think the results of this experimental cryoablation of normal lung tissue are reliable. Third, investigation concerning chronological changes and spatial distribution of temperature in the lung tissue during cryoablation was insufficient. The air in the bronchus and warm blood in the large vessels will disturb transbronchial cryoablation in the lungs. Simulation using gelatin and any artificial tube with thermal conductivity, like a bronchus or a vessel, may be useful in future experiments. Finally, the long-term influence of transbronchial cryoablation on the bronchus is unknown. The performance of animal experimentation is required before clinical application. However, the fact that the bronchus shows less susceptibility to freezing and maintained its structure with clinical percutaneous cryoablation is promising, although, in this case, the bronchus is frozen from the outside rather than intraluminally.

For future clinical application, the development of a cryoprobe specific for transbronchial insertion will be necessary, because all cryoprobes currently available for the treatment of malignancy are rigid and not suitable for orotracheal introduction into peripheral lung lesions. The expected characteristics that the transbronchial cryoprobe should meet are as follows: (1) flexibility of the part leading to the heat exchanger, which enables insertion orotracheally or through the working channel of bronchoscopy devices, allowing for easy introduction into a bronchus with a large turnout angle under bronchoscopic guidance, (2) small diameter of the heat exchanger, which enables easy perforation of the end of the conducting bronchus, (3) sufficient freezing ability, which provides a large central destruction zone less than $-20^{\circ} \mathrm{C}$ and (4) no risk of leakage of high pressure cooling gas or freezing agent outside of the probe, which might destroy non-malignant regions of the lungs or bronchi. When a probe dedicated for transbronchial cryoablation is developed in the future, a validation study for the safety and efficacy would be mandatory. The extension of the less than $-20^{\circ} \mathrm{C}$-thermal zone, which a miniaturised probe creates, must be measured. A bronchoscopic guidance system with endobronchial ultrasound or CT with an image reconstruction processing system is also necessary to guide the cryoprobe for adequate placement and for planning cryoablation.

Radiofrequency ablation may be another possible transbronchial modality for the treatment of small malignant lung lesions. It has been adopted percutaneously under CT guidance and is accompanied with complications such as pneumothorax, bronchopleural fistula and pleural effusion similar to percutaneous cryoablation. ${ }^{12}$ The risk of these complications also might be decreased via the use of the transbronchial approach. The development of a narrow electrode dedicated for the transbronchial approach and a validation study would be necessary before the clinical application of transbronchial radiofrequency ablation.

\section{CONCLUSION}

Transbronchial cryoablation with a rigid cryoprobe can effectively freeze peripheral lung tissue without any significant acute adverse effects. Our results suggest potential clinical application of transbronchial cryoablation for peripheral lung malignant lesions.

Acknowledgements The authors thank the Support Center for Medical Research and Education, Tokai University, for histological examination.

Contributors MKo, HY, SN, MKa and MI designed the research. MKo, RH, KO, HY, SN and MI performed the research. MKo analysed the data and wrote the paper.

Funding This study funded by Japan Society for the Promotion of Science (Grantin-aid for Scientific Research (C) \# 17K1080) 2016 Tokai University School of Medicine grant-in.

Competing Interest None declared.

Patient consent Not required.

Ethics approval statement All experiments were conducted in accordance with protocols approved by the Animal Experimentation Committee of Tokai University and was performed in compliance with its Animal Experimental Guidelines.

Provenance and peer review Not commissioned; externally peer reviewed. Data sharing statement No additional data are available.

Open access This is an open access article distributed in accordance with the Creative Commons Attribution Non Commercial (CC BY-NC 4.0) license, which permits others to distribute, remix, adapt, build upon this work non-commercially, and license their derivative works on different terms, provided the original work is properly cited, appropriate credit is given, any changes made indicated, and the use is non-commercial. See: http://creativecommons.org/licenses/by-nc/4.0/.

(C) Author(s) (or their employer(s)) 2018. Re-use permitted under CC BY-NC. No commercial re-use. See rights and permissions. Published by BMJ. 


\section{REFERENCES}

1. Kawamura M, Izumi Y, Tsukada N, et al. Percutaneous cryoablation of small pulmonary malignant tumors under computed tomographic guidance with local anesthesia for nonsurgical candidates. J Thorac Cardiovasc Surg 2006;131:1007-13.

2. Yamauchi $\mathrm{Y}$, Izumi $\mathrm{Y}$, Kawamura M, et al. Percutaneous cryoablation of pulmonary metastases from colorectal cancer. PLoS One 2011;6:e27086.

3. Yamauchi $\mathrm{Y}$, Izumi $\mathrm{Y}$, Hashimoto $\mathrm{K}$, et al. Percutaneous cryoablation for the treatment of medically inoperable stage I non-small cell lung cancer. PLoS One 2012; 7:e33223.

4. Inoue M, Nakatsuka S, Jinzaki M. Cryoablation of early-stage primary lung cancer. Biomed Res Int 2014;2014:1-8.

5. Yamauchi Y, Kawamura M, Inoue M, et al. [Results of percutaneous cryoablation for malignant lung tumors compared with radiotherapy] Kyobu Geka 2014:67:747-52.

6. Zhang X, Tian J, Zhao L, et al. CT-guided conformal cryoablation for peripheral NSCLC: initial experience. Eur J Radiol 2012;81:3354-62.
7. Pusceddu C, Sotgia B, Fele RM, et al. CT-guided thin needles percutaneous cryoablation (PCA) in patients with primary and secondary lung tumors: a preliminary experience. Eur J Radiol 2013;82:e246-e253.

8. Inoue M, Nakatsuka S, Yashiro $\mathrm{H}$, et al. Percutaneous cryoablation of lung tumors: feasibility and safety. J Vasc Interv Radiol 2012;23:295-302.

9. Yamauchi Y, Izumi Y, Hashimoto K, et al. Needle-tract seeding after percutaneous cryoablation for lung metastasis of colorectal cancer Ann Thorac Surg 2011;92:e69-e71.

10. Nakatsuka S, Yashiro $\mathrm{H}$, Inoue $\mathrm{M}$, et al. On freeze-thaw sequence of vital organ of assuming the cryoablation for malignant lung tumors by using cryoprobe as heat source. Cryobiology 2010;61:317-26.

11. Hashimoto K, Izumi Y, Yamauchi Y, et al. Prediction of the critical thermal zone during pulmonary cryoablation on computed tomography from correlated experimental and clinical findings. $J$ Thorac Cardiovasc Surg 2013;145:832-8.

12. Hiraki T, Gobara $\mathrm{H}$, Fujiwara $\mathrm{H}$, et al. Lung cancer ablation: complications. Semin Intervent Radiol 2013;30:169-75. 